\title{
Les paragonimoses humaine et expérimentales
}

\author{
Structure antigénique et relations hôte-parasite \\ de trois espèces du genre Paragonimus.
}

\author{
par A. CAPRON, A. VERNES, M. TSUJI et D. AFCHAIN
}

Laboratoire de Parasitologie de la Faculté de Médecine et de Pharmacie

(Directeur: Pr J. BIGUET), F 59 - Lille

Laboratoire de Parasitologie de l'Université de Chiba, Japon (Directeur: $\mathrm{P}^{r}$ YoKogawa)

\section{Résumé}

- L'analyse et la comparaison immunoélectrophorétiques de trois espèces du genre Paragonimus conduisent sur le plan taxinomique à une identité de vue parfaite avec la classification zoologique de ce genre.

— L'étude des réactions immunologiques de l'hôte vis-à-vis des trois espèces étudiées et celle des communautés antigéniques hôte-parasite, tout en confirmant la disparité de structure des trématodes, fait apparaître Paragonimus westermani comme une espèce adaptée aux félidés et secondairement à l'homme. Paragonimus ohirai semble au contraire se comporter comme une espèce étroitement adaptée aux mustélidés, alors que Paragonimus miyazakii serait une espèce en voie d'adaptation capable d'effectuer celle-ci à la fois chez les félidés et les mustélidés.

- Les résultats de l'analyse de 28 sérums de malades atteints de paragonimose à Paragonimus westermani, par différentes techniques immunologiques, démontrent la valeur de l'immunoélectrophorèse dans le diagnostic immunologique de la paragonimose.

* Travail effectué avec l'aide de l'Organisation Mondiale de la Santé et de l'Institut National de la Santé et de la Recherche Médicale. 


\section{Summary}

Comparative immunoelectrophoretical analysis between 3 Species of Paragonimus - at taxonomic level - lead to a perfect identity with the zoological classification of the genus.

Study of the host immunological reactions against these three species and of the antigenic communities between host and parasite confirms the disparity of structure of the Trematoda. It defines Paragonimus westermani as a species adopted to Felidae and secondarily to man. On the contrary, $P$. ohirai appears as a species strictly adapted to Mustelidae, while $P$. miyazakii seems in process of adaptation both to Felidae and Mustelidae.

Analysis of 28 sera coming from patients suffering from Paragonimosis westermani, and investigated by different immunological technics, proved the value of immunoelectrophoresis in immunological technics, proved the values of immunoelectrophoimmunological diagnosis of Paragonimosis.

\section{I. - INTRODUCTION}

Le genre Paragonimus, décrit pour la première fois en 1850 , de par la diversité des espèces qui le composent, la multiplicité des problèmes soulevés, en particulier taxinomiques et épidémiologiques, a suscité chez les chercheurs de tous horizons un intérêt sans cesse croissant.

Cet intérêt ne s'est pas démenti, et en particulier, ces quinze dernières années ont vu parallèlement à la découverte de nouvelles espèces, s'effectuer un très grand nombre de travaux consacrés à l'étude taxinomique du genre, à la biologie et l'épidémiologie des différentes espèces. En raison des incidences sur la pathologie humaine, notamment en ce qui concerne l'espèce Paragonimus westermani les réactions de l'hôte au parasitisme et en particulier la formation d'anticorps circulants ont été explorés par différentes méthodes immunologiques.

Depuis la description en 1850 par Diesing au Brésil, de Paragonimus rudis, isolé du poumon d'une loutre, 16 autres espèces de Paragonimus ont été décrites dont 5 ces dix dernières années. Un certain nombre d'entre elles apparaissent à la lumière des travaux modernes comme identiques à Paragonimus westermani.

Les récents travaux d'immunologie parasitaire de Biguet et coll. (9), Capron et coll. (1) mettaient en évidence :

- la disparité des structures antigéniques intergénériques caractérisant l'ordre des Trématodes, retrouvée même lorsque la comparaison antigénique était menée à l'intérieur du genre (Opistorchidés, Schistosomidés) ;

- la variabilité de la réponse immunologique de l'hôte, selon sa réceptivité vis-àvis du parasite en cause. 
Mis à part le problème d'ordre taxinomique d'intérêt purement zoologique, il semblait intéressant au vu des résultats obtenus de poursuivre plus avant l'étude de la structure antigénique et des relations hôte-parasite d'un genre de trématodes offrant à la fois une grande homogénéité phylétique et écologique et qui, tout en étant répandu assez largement à de nombreuses espèces animales, présentérait dans la gamme des animaux réceptifs le plus large éventail entre la réceptivité totale èt la résistance absolue.

Nous avons, pour ces raisons, choisi trois espèces différentes du genre Paragonimus offrant sur le plan biologique !es particularités précitées.

\section{II. - RAPPEL PARASITOLOGIQUE}

Les espèces du genre Paragonimus sont des trématodes de la famille des Troglotrématidés, parasites des bronches de nombreux mammifères parmi lesquels l'homme n'est pas exempt. Ils sont caractérisés par un corps très épais et la position latérale de l'utérus. Les métacercaires qui représentent la forme infectieuse pour l'hôte définitif sont enkystées chez des crustacés d'eau douce comme le crabe et l'écrevisse.

Le cycle évolutif est, à quelques variantes près, sensiblement le même pour toutés les espèces.

Les œufs sont éliminés dans la nature par l'intermédiaire des sel'es et des crachats. Ils s'embryonnent dans le milieu extérieur en deux ou trois semaines et les miracidiums qui s'en échappent doivent pour poursuivre leur évolution pénétrer chez le premier hôte intermédiaire, représenté par un mollusque aquatique ou amphibie. Chez celui-ci l'évolution qui dure environ dix semaines conduit le miracidium après passage par un stade rédie, puis la formation des rédies filles, au niveau de l'hépato-pancréas et grâce à un phénomène de polyembryonnie, à la production de nombreuses cercaires caractérisées par la présence d'un stylet antérieur.

Par ingestion ou par pénétration active, les cercaires s'enkystent chez un deuxième hôte intermédiaire, crustacé d'eau douce: crabe ou écrevisse. Les métacercaires ainsi formées évoluent en quelques semaines vers le stade inféctièux. On lés trouve principalement au niveau des branchies, du foie et des muscles de l'hôte intermédiaire. C'est par ingestion passive des crustacés infectés, orus ou mal cuits, que l'homme et de nombreux mammifères domestiques et sauvages contractent la maladie.

Chez l'hôte définitif les métacercaires se dékystent très rapidement dans l'intestin grêle et les jeunes vers traversent en quelques heures la paroi intestinale et le mésentère pour se retrouver dans la cavité abdominale. Après un séjour de dix à quinze jours dans celle-ci et quelquefois un court passage au niveau de la glande hépatique les vers traversent le diaphragme, la cavité pleurale et enfin le poumon pour aller se loger dans les bronches où ils vont tout en achevant leur maturation sexuelle s'entourer, souvent par paires, d'une réaction de l'hôte sous forme d'une fibrose kystique.

La durée de la maturation et la longévité des vers sont variables selon l'espèce en cause et selon l'hôte. 
La longévité peut atteindre pour Paragonimus westermani vingt ans en dehors de toute réinfection.

Si la localisation pulmonaire est de règle, on observe cependant et avec une fréquence non négligeable des localisations erratiques, en particulier au niveau du système nerveux central.

Présents en Afrique centrale (26) et en Amérique du Sud les Paragonimus sont surtout rencontrés en Asie de l'Est et particulièrement en Corée, au Japon, à Formose, en Chine centrale et aux Philippines. Ceci explique dans une grandè mesure l'intérêt porté à ce problème par les chercheurs asiatiques et tout spécialement japonais.

En ce qui concerne les trois espèces étudiées dans ce travail, les cycles évolutifs se caractérisent comme suit :

\section{Paragonimus westermani (Kerbert, 1878), Braun, 1899.}

Le premier hôte intermédiaire est un moliusque operculé du genre Semisulcospira. L'espèce $S$. libertina est la plus fréquemment incriminée. L'évolution chez le mollusque est de neuf semaines.

De nombreuses espèces de crabes d'eau douce et d'écrevisses sont susceptibles de jouer le rôle de deuxième hôte intermédiaire. Eriocheir japonicus et Eriocheir sinensis ainsi que Potamon dehaani sont les espèces le plus souvent en cause. Chez éux, la métacercaire devient infectieuse dans un laps de temps variant de quatre à cinq semaines.

Parasite fréquent de l'homme, Paragonimus westermani est habituellement rencontré chez les félidés, y compris les grandes espèces sauvages, ainsi que chez le porc et le chien qui jouent ainsi le rôle important de réservoir de virus.

Chez l'homme la maturité sexuelle est atteinte en soixante-dix jours. La longévité des vers peut atteindre vingt ans.

\section{Paragonimus obirai Miyazaki, 1939.}

Assiminea parasitologica et Paludinella devilis sont les premiers hôtes intermédiaires le plus généralement rencontrés. L'évolution du parasite chez eux est de 120 à 135 jours et les cercaires libérées pénètrent activement le deuxième hôte intermédiaire, un crabe d'eau douce: Sesarma dehaani ou Sesarma intermedia. Chez celui-ci, les métacercaires infectieuses sont surtout présentes au niveau du foie et plus rarement au niveau des muscles.

De nombreux petits mammifères sauvages de la famille des mustélidés, comme le blaireau et la belette sont trouvés porteurs de cette espèce dans la nature. Le rat, le porc, le sanglier et le chien jouent également le rôle de réservoir de virus.

Chez le rat expérimentalement infecté, les vers atteignent leur maturité sexuelle en trente jours. La durée de vie de $P$. ohirai semble beaucoup plus brève que pour $P$. westermani; les vers commencent à mourir dès le sixième mois d'évolution. 
Рното 1. - Immunoélectrophorégramme de l'antigène Paragonimus westermani révélé par un immunsérum de lapin anti Paragonimus westermani

Pното 2. - Immunoélectrophorégramme de l'antigène Paragonimus ohirai révélé par un inmunsérum de lapin anti Paragonimus ohirai

Рното 3. - Immunoélectrophorégramme de l'antigène Paragonimus miyazakii révélé par un immunsérum de lapin anti Paraganimus miyazakii

Pното 4. - Communautés antigéniques des trois espèces du genre Paragonimus, observées en immunoélectrophorèse. Les trois antigènes sont révélés par un immunsérum de lapin anti Paragonimus westermani

Рното 5. - Immunoélectrophorégramme de l'antigène Paragonimus westermani révélé par deux sérums de malades atteints de paragonimose à Paragonimus westermani
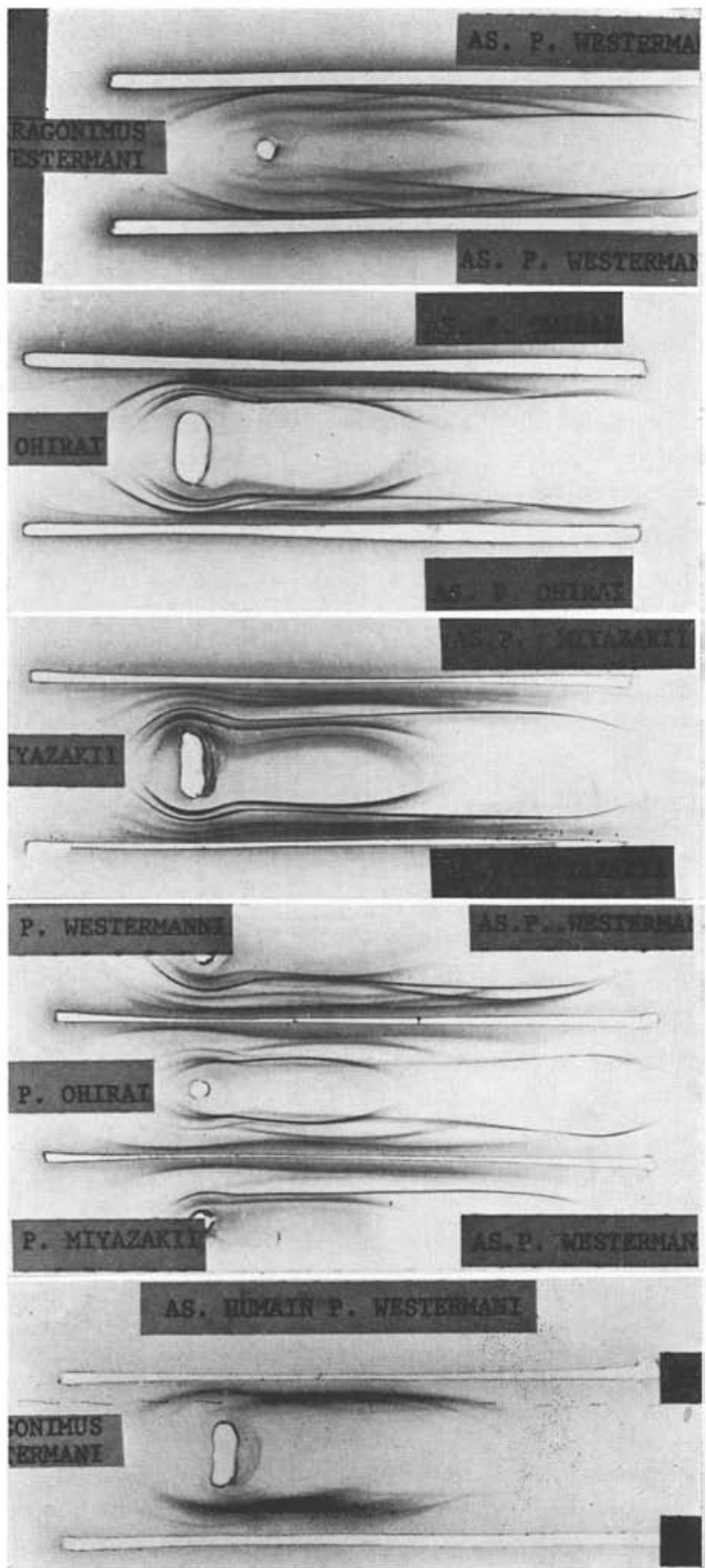


\section{Paragonimus miyazakii Kamo et coll., 1961.}

Cette espèce décrite plus récemment a pour premier hôte intermédiaire Bythinella (Moria) nipponica et pour deuxième hôte intermédiaire un crabe d'eau douce Potamon dehaani. L'hôte définitif est le chat, mais aussi le rat.

\section{Différenciation des espèces:}

En dehors de l'étude des hôtes réceptifs tant intermédiaires que définitifs, l'observation morphologique permet d'aboutir à la classification des différentes espèces. Celle-ci est conduite sur les différentes formes larvaires et adultes.

\section{a) Cercaires.}

Il s'agit toujours de xiphidiocercaires microcerques. C'est surtout par le compte des cellules flammes et l'établissement de leur formule que l'on obtient des renseignements utiles. La formule pour $P$. westermani est

et pour $P$. ohirai

$$
2[(3+3+3+3+3)+(3+3+3+3+3)]=60
$$

$$
2[(1+1+1+1)+(1+1+1+1)]=16
$$

\section{b) Métacercaires.}

Elles sont de taille et de forme variables selon l'espèce. Celles de $P$. miyazakit sont sphériques, possédant une membrane externe fine et une membrane interne épaisse. Leur diamètre varie de 400 à $500 \mu$.

Les métacercaires de $P$. westermani sont plus petites mesurant de 340 à $450 \mu$ de diamètre.

Par contre, les métacercaires de $P$. ohirai sont ovalaires mesurant de 260 à $350 \mu$ sur 300 à $350 \mu$.

\section{c) Adultes.}

Les Paragonimus sont des vers trématodes de petite taille et très épais. Les mesures moyennes sont pour l'espèce $P$. westermani de $9,6 \mathrm{~mm}$ de long sur $5 \mathrm{~mm}$ de large et $3,7 \mathrm{~mm}$ d'épaisseur.

Le diamètre comparé des ventouses orales et ventrales permet une première dis. tinction.

Dans le groupe qui nous intéresse et où le diamètre de la ventouse orale est inférieur à celui de la ventouse ventrale, l'étude de la forme et du groupement des épines cuticulaires ainsi que celle de la conformation de l'ovaire permettént de distinguer quatre sous-groupes: les espèces qui nous intéressent ici font partie de trois d'entre eux. 


\section{d) Les cufs.}

Operculés et non embryonnés à la ponte, les œufs de Paragonimus sont de forme et de taille variables selon l'espèce en cause.

La longueur varie de 60 à $100 \mu$ et la largeur de 40 à $70 \mu$.

\section{III. - ANALYSE DES TRAVAUX ANTERIEURS}

Les premières observations immunologiques en matière de paragonimose ont été rapportées par Ando en 1917 (6). Elles concernaient l'étude de sérums de malades porteurs de $P$. westermani, par la réaction de fixation du complément utilisant un antigène extrait des vers frais par une solution salée et alcoolique. Cet auteur signalait dans son travail l'existence de réactions faussement positives.

A ce travail a fait suite une importante série de publications sur ce sujet. La plupart tendent à la mise en évidence, par réaction de fixation du complément ou par intradermo-réaction, des anticorps présents dans les sérums de malades ou d'animaux expérimentalement infectés. Nous analyserons les plus marquants d'entre eux dans la première partie.

Un nombre restreint de travaux plus récents et dépassant le seul aspect pratique nous apporte des renseignements sur la structure protéique et antigénique des Paragonimus ainsi que sur les relations hôte-parasite des paragonimoses. Nous les relaterons dans une deuxième partie.

\section{A. - Anticorps circulants et paragonimose}

Yokogawa S. et coll. (3), Kent (2) et Yokogawa M. (4) ont dans d'excellentes revues générales fait le point sur les nombreux résultats obtenus par une gamme très variée de recherches sérologiques dans les paragonimoses humaines et expérimentales.

Manoji (15) utilisant le «ring test » et un antigène extrait de $P$. westermani adultes par des solutions salines observe sur un petit nombre de sérums de malades et sur celui d'un chien expérimentalement infecté des réactions positives à des dilutions sériques variant du $1 / 200^{\circ}$ au $1 / 800^{\circ}$. Manoji signale des réactions croisées avec l'antigène extrait de $P$. ohirai.

So (21) utilisant également le « ring test » rapporte les premières observations immunologiques dans la paragonimose expérimentale à $P$. ohirai avec des conclusions semblables.

L'on doit à Yokogawa M. et Awano (25), Sadun et coll. (18) les premiers travaux sérologiques d'importance sur la paragonimose humaine et expérimentale à $P$. westermani.

Yokogawa étudiant, par la réaction de fixation du complément lue au point $50 \%$ d'hémolyse et le test de floculation de particules de bentonite, une série de Annales de Parasitologie, humaine et comparée (Paris), t. 44, 1969, $\mathrm{n}^{\circ} 6$ 
69 malades confirmés parasitologiquement, observe avec un antigène extrait de vers adultes dans un tampon véronal, 56 réactions positives à des taux significatifs. La dilution antigénique est de l'ordre du $1 / 5000^{\circ}$.

Ces auteurs relatent parallèlement une étude comparative détaillée des résultats obtenus par les réactions sérologiques et l'intradermoréaction.

L'utilisation des techniques d'immunodiffusion est rapportée pour la première fois par Phillipson et coll. (17). Les auteurs ayant réalisé l'infection expérimentale de chats par Clonorchis sinensis, Opistorchis felineus, Paragonimus westermani et Paragonimus kellicotti mettent en évidence en double diffusion en gélose les communautés antigéniques existant entre ces différentes espèces.

Sawada et coll. (19 $a$ et $19 b$ ) dans un travail sur le diagnostic immunologique de la paragonimose étudient par des méthodes physicochimiques les antigènes solubles de Paragonimus westermani extraits par sonnage et broyage dans de l'eau distillée des vers fraîchement récoltés. L'électrophorèse de zone sur papier permet d'isoler trois fractions protéiques: négative, neutre et positive. La fraction neutre donne, pour ces auteurs, les meilleurs résultats, dans la recherche par le « ring test » des anticops de trois malades et de trois chiens expérimentalement infectés.

Dans la deuxième partie de leur travail les mêmes auteurs, à l'aide des techniques d'électrophorèse en gel d'agarose et de la chromatographie sur carboxyméthylcellulose et sur DEAE poursuivent plus avant l'étude des mêmes antigènes. Six fractions sont séparées sur carboxyméthylcellulose dont une dénommée CM 1 qui peut être séparée elle-même après chromatographie sur DEAE cellulose en sept sous-fractions. L'une d'entre elles, la fraction $\mathrm{C}_{1} \mathrm{SS}$ soluble après centrifugation, mais précipitable par la dihydrostreptomycine, s'avère particulièrement active dans les tests cutanés pratiqués sur des malades atteints de paragonimose; elle développe chez eux dés réactions positives à la dose de $0,01 \mu \mathrm{g}$.

Récemment Tada (22) au cours d'une étude physiologique et sérologique dans le temps de la paragonimose expérimentale du rat à $P$. miyazakii rapporte les résultats suivants :

- L'activité transaminasique des sérums de rats infectés est augmentée de façon constante; les transaminases glutamo-pyruviques (S.G.P.T.) atteignent rapidement, au $30^{\circ}$ jour, un taux maximum qui est de 130 U.I.T., puis décroissent. Par contre, les transaminases glutamo-oxaliques (S.G.O.T.) ont une élévation progressive pour atteindre 130 U.I.T. vers le $70^{\circ}$ jour suivant l'infection alors que les S.G.P.T. sont revenues à un taux normal ou subnormal.

Parallèlement, l'électrophorèse sur papier des sérums de rats parasités révèle dès le $20^{\circ}$ jour, une diminution de la sérumalbumine avec augmentation concomitante des gamma-globulines.

L'étude des mêmes sérums par double diffusion en gel d'agarose et à l'aide d'un antigène soluble, extrait des vers adultes en tampon phosphate $\mathrm{pH} 7,4$ met en évidence une apparition d'anticorps précipitants dès le $20^{\circ}$ jour, atteignant leur taux maximal au $90^{\circ}$ jour pour décrôtre ensuite jusqu'au $150^{\circ}$ jour. 
Au $90^{\circ}$ jour, six systèmes précipitants antigènes-anticorps peuvent être ainsi isolés pour un des animaux d'expérience.

Seed et col. (20) ont mené sur les sérums de chats expérimentalement infectés par $P$. kellicotti, une série d'investigations sérologiques et immuno'ogiques. Les antigènes proviennent de $P$. kellicotti adultes et sont extraits dans un tampon borate à $\mathrm{pH} 8,5$.

Ces auteurs obtiennent des résultats variables selon la technique utilisée; les meilleurs résultats sont obtenus avec la réaction de fixation du complément (dilutions positives allant du 1/4 au 1/4096) et la technique d'Ouchterlony (trois sérums négatifs sur quatorze testés; un sérum donnait avec l'antigène cinq arcs de précipitation). Par contre, la recherche des anticorps par l'intradermoréaction, l'anaphylaxie générale et le test d'immobilisation miracidien se révélait décevante.

\section{B. - L'analyse immunoélectrophorétique des antigènes d'Helminthes. Répercussions sur la connaissance des relations hôte-parasite et sur celle des Paragonimus}

Les techniques immunoélectrophorétiques de Grabar et Williams (12, 13), ont été appliquées par Biguet et coll. $(7,8,9)$ à l'étude des problèmes d'immunologie parasitaire. Technique douée d'un grand pouvoir analytique, l'immunoélectrophorèse a permis de «disséquer» la mosaïque antigénique des helminthes et des champignons et d'isoler ainsi certaines fractions protéiques remarquables, spécifiques de chacune des espèces de parasites analysés.

Utilisant cette technique Capron et coll. (10) ont étudié les sérums de 57 malades atteints ou suspects de paragonimose à $P$. westermani. Les antigènes de $P$. westermani sont extraits dans une solution saline de $\mathrm{C} 1 \mathrm{Na} 0,017 \mathrm{M}$ après broyage au mortier glace.

L'immunoélectrophorèse se révèle être une méthode excellente pour le diagnostic de cette parasitose.

Deux à sept systèmes précipitants sont mis en évidence dans les sérums des malades confirmés parasitologiquement et dans un deuxième groupe de malades présentant une réaction de fixation du complément positive.

Parmi ces systèmes précipitants, l'un d'entre eux dénommé arc 1 de l'immunoélectrophorégramme présente des particularités remarquables. Il apparaît précocément lors de l'infection expérimentale de l'animal et l'immunisation du lapin. Il ne se retrouve pas dans d'autres helminthiases et n'est pas révélé par les antigènes d'autres espèces d'helminthes. Sa présence sur l'immunoélectrophorégramme confirme à elle seule le diagnostic immunologique de paragonimose.

Il existe également des réactions croisées intéressant d'autres fractions de la mosaïque antigénique de $P$. westermani; elles sont nettes avec lès autres antigènes 
de trématodes utilisés dans ce travail: Fasciola hepatica, Dicrocoelium dendriticum et Opistorchis felineus.

Sensiblement à la même époque, des résultats analogues sont obtenus par deux autres équipes de chercheurs. Yogore et coll. (24) mettent en évidence par immunoélectrophorèse deux à cinq systèmes précipitants dans les sérums de quatres malades et de six chats expérimentalement infectés par $P$. westermani. Deux d'entrè eux sont présents dans tous les sérums humains étudiés. L'équipe de chercheurs du 406th Medical Laboratory (14) observe également par immunoélectrophorèse cinq systèmes précipitants dans les sérums de lapins immunisés par voie intraveineuse par un antigène de $P$. westermani extrait des adultes par une solution de $\mathrm{C} 1 \mathrm{Na}$ à $0,9 \%$ après broyage.

Ce travail est complété par une comparaison des antigènes solubles de $P$. westermani et de $P$. ohirai à l'aide de l'électrophorèse en gel d'acrylamide. Vingt-deux fractions sont ainsi identifiées pour chaque espèce ; elles diffèrent sensiblement en position et en intensité.

Des résultats originaux sont rapportés par Tran Van Ky et coll. (23) qui utilisant les techniques d'enzymologie appliquées à l'immunoélectrophorèse, isolent dans la mosaïque antigénique de $P$. westermani 14 activités enzymatiques différentes.

Dans une étude récente de la structure antigénique de 30 espèces d'helminthes parasites et de 17 espèces animales hôtes intermédiaires et définitifs (verrtébrés et invertébrés), Capron et coll. (1) apportent des conclusions intéressant les relations hôte-parasite au cours des Paragonimoses humaines et expérimentales.

L'analyse immunoélectrophorétique des antigènes helminthiques permet d'identifier 19 à 25 fractions différentes. Vingt fractions sont mises en évidence pour $P$. westermani.

La comparaison immunoélectrophorétique des Trématodes objective une disparité de structure intergénérique, au contraire de ce qui est observé chez les Nématodes et surtout chez les Cestodes. Cependant cette disparité s'atténue lors des comparaisons intragénériques en particulier chez les Opistorchidés et les Schistosomidés. savoir :

L'analyse des relations hôte-parasite permet de confirmer certains faits, à

- Il existe un rapport généralement étroit entre les réactions immunologiques et le degré d'adaptation à un hôte considéré. Ce fait est bien mis en évidence dans les distomatoses et en particulier dans les paragonimoses humaine et expérimentale à $P$. westermani, de même que dans la paragonimose expérimentale à $P$. ohirai.

- Les communautés antigéniques unissant le parasite à l'hôte, déjà observées chez les schistosomes (11), sont décrites et interprétées comme un mode d'expréssion de l'adaptation parasitaire.

L'étude des relations hôte-parasite dans les paragonimoses démontre l'existence de communautés antigéniques importantes entre les Paragonimus et leur hôte définitif d'une part, $P$. westermani et son hôte intermédiaire d'autre part. Ces communautés 
antigéniques sont d'autant plus importantes que l'adaptation parasitaire est la meilleure possible.

Une théorie suggérant l'intervention de phénomènes d'induction et permettant d'expliquer les différents phénomènes observés est proposée.

\section{IV. - MATERIEL ET METHODES}

\section{1) Métacercaires infectieuses.}

Les métacercaires infectieuses de $P$. westermani ont été recueillies sur Eriocheir japonicus, celles de $P$. ohirai sur Sesarma dehaani et sur Potamon dehaani pour P. miyazakii.

Les métacercaires peuvent être conservées pendant plusieurs jours à $4^{\circ} \mathrm{C}$ dans de l'eau douce ou physiologique.

A notre connaissance, c'est la première fois en France qu'ont été obtenus chez l'animal d'expérience des exemplaires vivants des trois espèces étudiées.

\section{2) Animaux d'expérience.}

Le chat domestique a été utilisé pour l'obtention des adultes de $P$. westermani et de $P$. miyazakii. Le rat blanc de souche Wistar est particulièrement réceptif à l'espèce $P$. ohirai. On peut également l'utiliser pour l'infection par les deux autres espèces. Dix chats ont été infectés: six avec $P$. westermani, quatre avec $P$. miyazakii. Chaque chat a reçu une dose de 50 métacercaires infectieuses. Les animaux sont sacrifiés entre le $90^{\circ}$ et le $140^{\circ}$ jour pour $P$. westermani et du $50^{\circ}$ au $70^{\circ}$ jour pour $P$. miyazakii.

Neuf rats ont été soumis à l'infection : cinq avec $P$. ohirai, deux avec $P$. westermani, deux avec $P$. miyazakii. Chacun d'entre eux a reçu une dose de 15 métacercaires infectieuses.

L'infection expérimentale de l'animal est réalisée par gavage, à l'aide d'une pipette Pasteur contenant les métacercaires dans une petite quantité de sérum physiologique salé à $9 \%$, après avoir vérifié à la loupe binoculaire la vitalité de ces dernières.

Il faut veiller au cours du prélèvement des métacercaires à ne pas provoquer leur dékystement par une aspiration trop brutale.

\section{3) Sérums.}

a) Les sérums des animaux infectés ont été prélevés à un rythme bi-mensuel.

Ceux des chats sont prélevés à la seringue par ponction veineuse au niveau de la veine radiale. Il est indispensable d'être secondé par un aide qui maintient la tête de l'animal emprisonnée dans une toile de forte épaisseur.

En ce qui concerne les rats, la ponction se fait à l'aide d'une pipette Pasteur stérile au niveau du sinus veineux de l'œil, le sang montant dans la pipette sous l'effet dé la pression veineuse ou par aspiration de l'opérateur à l'aide d'un tuyau de latex adapté 
à l'extrémité supérieure de la pipette. Il faut signaler la particulière fragilité de la paroi nasale osseuse du rat qui sans précaution sera fracturée, entraînant la mort de l'animal par hémorragie dans un grand nombre de cas.

b) Nous avons disposé par ailleurs des sérums de 28 malades, tous confirmés par l'examen parasitologique.

c) Immunsérums de lapins.

Des immunsérums de lapins furent préparés pour chacune des trois espèces, par injection hebdomadaire sous-cutanée de $20 \mathrm{mg}$ d'antigène brut incorporé dans un volume égal d'adjuvant de Freund.

Un lapin fut immunisé pour chaque espèce de Paragonimus. Les sérums sont prélevés deux fois par mois à la veine de l'oreille par aspiration sous vide. Ils sont testés en immunoélectrophorèse vis-à-vis de l'antigène correspondant jusqu'à l'obtention du nombre maximal de précipitines.

Nous avons pu également utiliser les sérums de quatre autres lapins immunisés vis-à-vis de $P$. westermani dans une précédente expérimentation. Pour l'étude des réactions croisées avec les autres helminthes, nous avons utilisé les immunsérums préparés au laboratoire au cours de précédentes recherches.

d) Saturation des immunsérums.

Afin de déterminer avec certitude les communautés antigéniques des trois espècés en cause les immunsérums ont été saturés par les antigènes hétérologues, puis appliqués ensuite contre les différents antigènes des trois espèces.

La saturation des antisérums bruts est généralement réalisée par l'adjonction dans $1 \mathrm{ml}$ de sérum de 10 à $20 \mathrm{mg}$ d'antigène standard ; elle est conduite d'abord à $37^{\circ} \mathrm{C}$ pendant 1 heure, puis pendant une nuit à $4^{\circ} \mathrm{C}$. Seul le surnageant prélevé après centrifugation est recueilli.

\section{4) Antigènes.}

\section{a) ANTigènes BRUts.}

Les vers sont recueillis après sacrifice de !'animal et ouverture des kystes. Ils sont lavés à plusieurs reprises dans du sérum physiologique, puis une dernière fois dans de l'eau distillée avant d'être lyophilisés.

Ces premières opérations conduisent à l'obtention de l'antigène brut utilisé pour l'immunisation des lapins.

\section{b) ANTigènes Solubles Standards.}

$\alpha$ Antigène somatique.

Il est extrait par broyage mécanique au mortier glace de l'antigène brut dans une solution de chlorure de sodium 0,017 M.

Après 8 à 10 broyages successifs, le liquide obtenu est centrifugé à $4^{\circ} \mathrm{C}$, à 20.000 
tours par minute pendant une heure. Le surnageant recueilli est dialysé en boudin de cellophane pendant 48 heures contre l'eau distillée et enfin lyophilisé.

Au broyage par mortier glace peut être substituée une technique consistant à broyer les vers prélevés faîchement dans une solution de $\mathrm{CINa}$ à $0,017 \mathrm{M}$, à l'aide d'un broyeur électrique à couteau et ce à une température proche de $0^{\circ} \mathrm{C}$. La solution ainsi obtenue est ensuite traitée comme précédemment. Les antigènes obtenus par cette variante sont d'une qualité équivalente.

B) Antigènes métaboliques.

Par incubation des vers dans une petite quantité d'eau distillée, on obtient au bout d'une heure un liquide fortement coloré qui, après dialyse et lyophilisation, conduit à l'antigène métabolique.

\section{5) Techniques.}

a) Double DifFusion EN Gel D'AGarose.

Nous avons utilisé la technique d'Ouchterlony (16) modifiée par Abelev et Zvetkov (5) pour déterminer le meilleur rapport antigène-anticorps.

b) IMMUNOÉLECTROPHORÈSE.

La technique utilisée est celle de Grabar et Williams $(12,13)$ et adaptée par Biguet et coll. $(7,8,9)$.

Le gel est préparé à raison de $90 \mathrm{mg}$ d'agarose purifiée dans $10 \mathrm{~cm}^{3}$ de tampon véronal $\mathrm{pH} 8,2$.

Après électrophorèse préparative de l'antigène à la concentration de $20 \mathrm{mg}$ pour $0,1 \mathrm{ml}$ d'eau distillée, les immunoélectrophorégrammes sont révélés par les immunsérums concentrés au 1/3 par lyophilisation.

Les lames laissées en incubation à la température du laboratoire et en atmosphère humide la première nuit sont conservées ensuite 48 heures à $4^{\circ} \mathrm{C}$.

Après lavage et déminéralisation, elles sont séchées et colorées à l'amidoschwarz et enfin différenciées dans plusieurs solutions d'eau acétifiée.

c) RÉACTION DE FIXATION DU COMPLÉmENT.

Les sérums ont été techniqués par la méthode de Kolmer et lecture au point $100 \%$ d'hémolyse.

\section{V. - RESULTATS}

\section{A. - Structure antigénique des trois espèces.}

\section{1) Antigènes somatiques.}

L'analyse immunoélectrophorétique des antigènes des trois espèces de Paragonimus par les immunsérums homologues de lapin permet de révéler un minimum de 19 à 
23 fractions antigéniques distinctes pour chacune des trois espèces (Tableau A - Photos 1, 2, 3).

\section{Tableau A}

Antigènes somatiques. Structure et communautés antigénique des Paragonimus (Nombre minimal de fractions observées en immunoélectrophorèse)

\begin{tabular}{|c|c|c|c|}
\hline ANTIGÈNES & $\begin{array}{l}\text { ANTISÉRUM DE } \\
P \text {. westermani }\end{array}$ & $\begin{array}{l}\text { ANTISÉRUM DE } \\
P . \text { miyazakii }\end{array}$ & $\begin{array}{c}\text { ANTISÉRUM DE } \\
P \text {. ohirai }\end{array}$ \\
\hline P. westermani .......... & 23 & 14 & 14 \\
\hline P. miyazakii ............ & 14 & 19 & 13 \\
\hline P. ohirai $\ldots \ldots \ldots \ldots \ldots$ & 14 & 13 & 19 \\
\hline
\end{tabular}

2) ANTigènes métaboliques.

Dans les mêmes conditions, l'immunoélectrophorèse des antigènes métaboliques permet de mettre en évidence 8 à 11 fractions antigéniques (Tableau B).

\section{B. - Structure antigénique comparée des trois espèces.}

Les réactions croisées observées en immunoélectrophorèse intéressent un peu plus de la moitié de la structure antigénique totale de chaque couple considéré (tableau A et $\mathrm{B}$, photo 4).

La comparaison des index d'isologie montre des communautés antigéniques légèrement supérieures entre $P$. miyazakii et $P$. ohirai (Index $=0,68$ ) qu'entre ces deux espèces et $P$. westermani (index $=0,61$ ). Cette observation est confirmée par l'étude des fractions spécifiques de chaque espèce après saturation des immunsérums par l'antigène hétérologue (tableau $\mathrm{C}$ ).

Sept à huit fractions antigéniques de $P$. westermani ne sont pas partagées avec les deux autres espèces.

Trois fractions spécifiques seulement ont pu être isolées de $P$. miyazakii et de $P$. ohirai par saturation croisée èntre les deux espèces.

La fraction 1 de $P$. westermani (la plus proche du pôle négatif de l'immunoélectrophorégramme) décrite par Capron et coll. (10) est partagée par les trois espèces, Elle n'est pas intéressée par les réactions croisées avec les autres helminthes.

\section{C. - Communautés antigéniques du genre Paragonimus avec les helminthes.}

Le tableau D résume les résultats obtenus. 
Les communautés antigéniques avec les Trématodes varient de deux à six fractions, généralement quatre à cinq, ce qui représente la communauté antigénique moyenne observée entre tous les Trématodes.

Avec les Cestodes, une à deux fractions seulement sont intéressées et en ce qui concerne les Nématodes, trois fractions sont communes avec Dirofilaria immitis, filaroidea parasite du chien.

\section{Tableau B}

ANtigÈnes métaboliques. Structure et communautés antigéniques des Paragonimus (Nombre minimal de fractions observées en immunoélectrophorèse)

\begin{tabular}{|c|c|c|c|}
\hline ANTIGÈNES & $\begin{array}{l}\text { ANTISÉRUM DE } \\
P \text {. westermani }\end{array}$ & $\begin{array}{l}\text { ANTISÉRUM DE } \\
P . \text { miyazakii }\end{array}$ & $\begin{array}{l}\text { ANTISÉRUM DE } \\
P \text {. ohirai }\end{array}$ \\
\hline Inc. P. westermani ...... & 10 & 5 & 6 \\
\hline Inc. P. miyazakii $\ldots . . \ldots$ & 5 & 8 & 5 \\
\hline Inc. $P$. ohirai $\ldots$ & 6 & 5 & 11 \\
\hline
\end{tabular}

Tableau C

NOMBRE DE FRACTIONS SPÉCIFIQUES D'UNE ESPÈCE VIS-A-VIS D'UNE AUTRE ESPÈCE, ISOLÉES APRÈS SATURATION DES IMMUNSÉRUMS CORRESPONDANTS (IMMUNOÉLECTROPHORÈSE)

\begin{tabular}{|c|c|c|c|}
\hline & P. westermani & P. miyazakii & P. ohirai \\
\hline P. westermani $\ldots \ldots \ldots$ & & 8 & 7 \\
\hline P. miyazakii ........... & 8 & & 3 \\
\hline P. ohirai ... & 7 & 3 & \\
\hline
\end{tabular}

\section{D. - Relations hôte-parasite.}

1. - La réponse de l'hôte au parasitisme. Apparition et évolution des anticorps précipitants.

\section{a) Paragonimose expérimentale.}

Les tableaux $\mathrm{E}$ et $\mathrm{F}$ synthétisent les résultats acquis par les techniques de double diffusion en agarose et l'immunoélectrophorèse.

a) Paragonimose expérimentale à $P$. westermani.

Chez le chat, les anticorps précipitants apparaissent à la cinquième semaine. Leur taux s'élève très progressivement et lentement. Trois à cinq systèmes précipitants sont révélés dès la dixième semaine d'évolution par la technique d'Ouchterlony. 


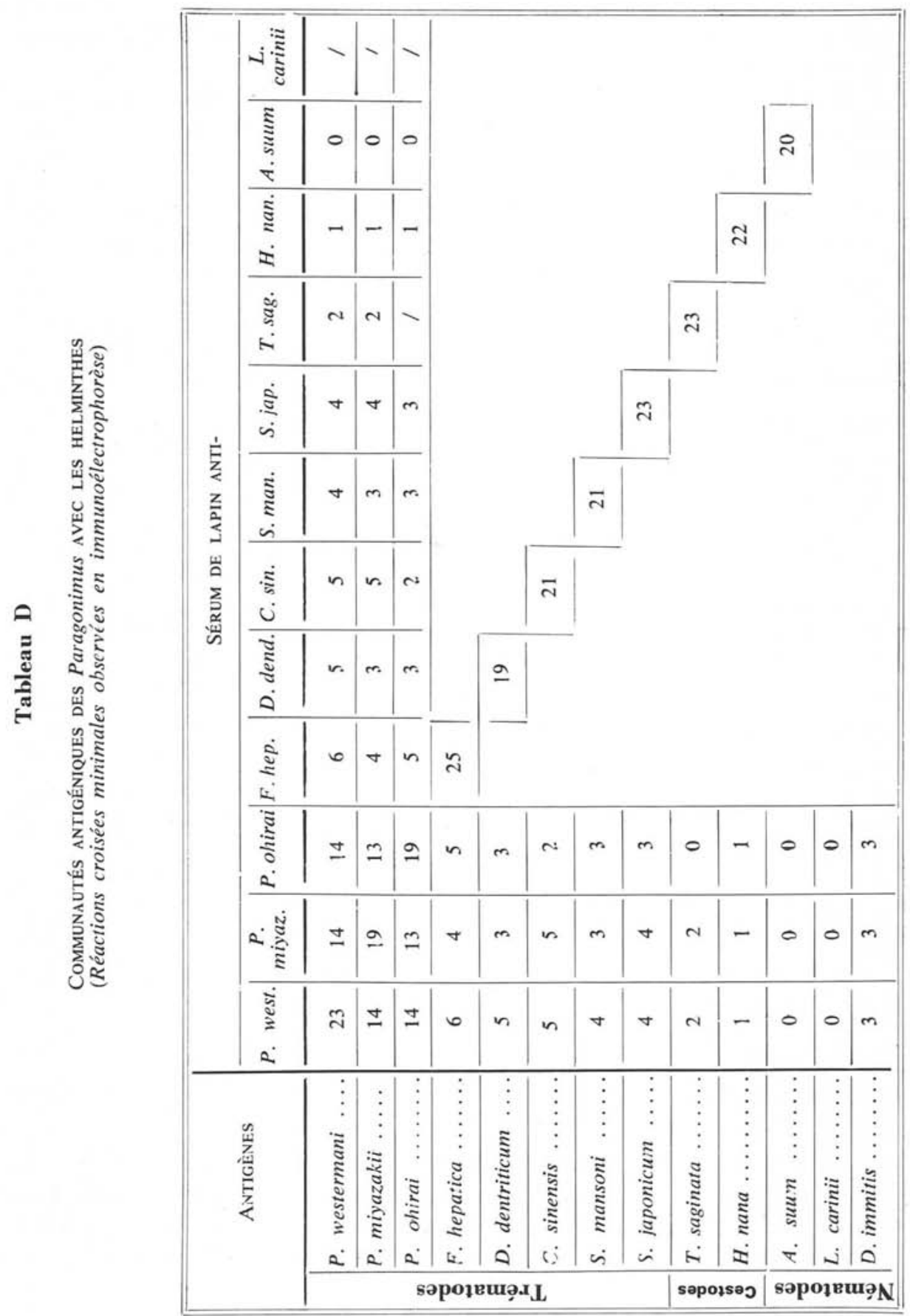


L'immunoélectrophorèse permet de mettre en évidence dès l'apparition des premiers anticorps, et de façon constante, la fraction 1.

Le maximum de systèmes précipitants est observé en immunoélectrophorèse dès la dixième semaine ; il varie de quatre à neuf selon les animaux, le nombre moyen maximal étant de 5,8.

La moitié environ de ces systèmes précipitants peuvent être révélés par les antigènes hétérologues de $P$. miyazakii et $P$. ohirai.

L'infection expérimentale du rat est constamment et immédiatement abortive. Aucun anticorps précipitant n'a pu être décelé dans les sérums de deux rats ayant absorbé des métacercaires infectieuses de $P$. westermani.

ß) Paragonimose expérimentale à $P$. miyazakii.

L'apparition des anticorps précipitants chez le chat expérimentalement infecté est plus précoce, dès la troisième semaine ; il en va de même chez le rat.

L'évolution de ces anticorps est lentement progressive.

Le maximum de systèmes précipitants révélés en immunoélectrophorèse est de sept ; le nombre maximal moyen est de quatre.

Ici encore d'intenses réactions croisées sont observées avec les antigènes des deux autres espèces.

L'infection du rat qui se réalise facilement n'aboutit qu'à la formation de deux systèmes précipitants du moins jusqu'à la sixième semaine. Leur apparition est précoce.

\%) Paragonimose expérimentale à $P$. ohirai.

L'étude des sérums de rats expérimentalement infectés permet de noter l'apparition très précoce des anticorps précipitants dès la première semaine suivant l'infection. Ceci est vraisemblablement en rapport avec la maturation très rapide de cette espèce.

L'évolution de ces anticorps est progressive et le maximum est rapidement atteint.

Le nombre maximal observable en immunoélectrophorèse est de six systèmes précipitants : le nombre moyen maximal est 4,6 .

Les réactions croisées avec les deux autres espèces sont très nettes.

b) Paragonimose humaine a $P$. westermani.

Les résultats immunologiques des sérums de 28 malades sont rassemblés dans le tableau $G$ dont l'analyse permet de retenir les points suivants :

- tous les sérums étudiés sont positifs en immunoélectrophorèse avec présence constante de la fraction 1 (photo 5). Le nombre de systèmes précipitants révélés varie de deux à douze avec un chiffre moyen maximal de cinq.

- la réaction de fixation du complément dont les taux ne peuvent être interprétés de façon significative qu'à partir de la dilution du $1 / 20^{\circ}$ permet $85 \%$ de succès.

- des réactions croisées sont également observées avec les antigènes des deux autres espèces.

- l'intradermoréaction se révèle être également une technique très utile et efficace. 


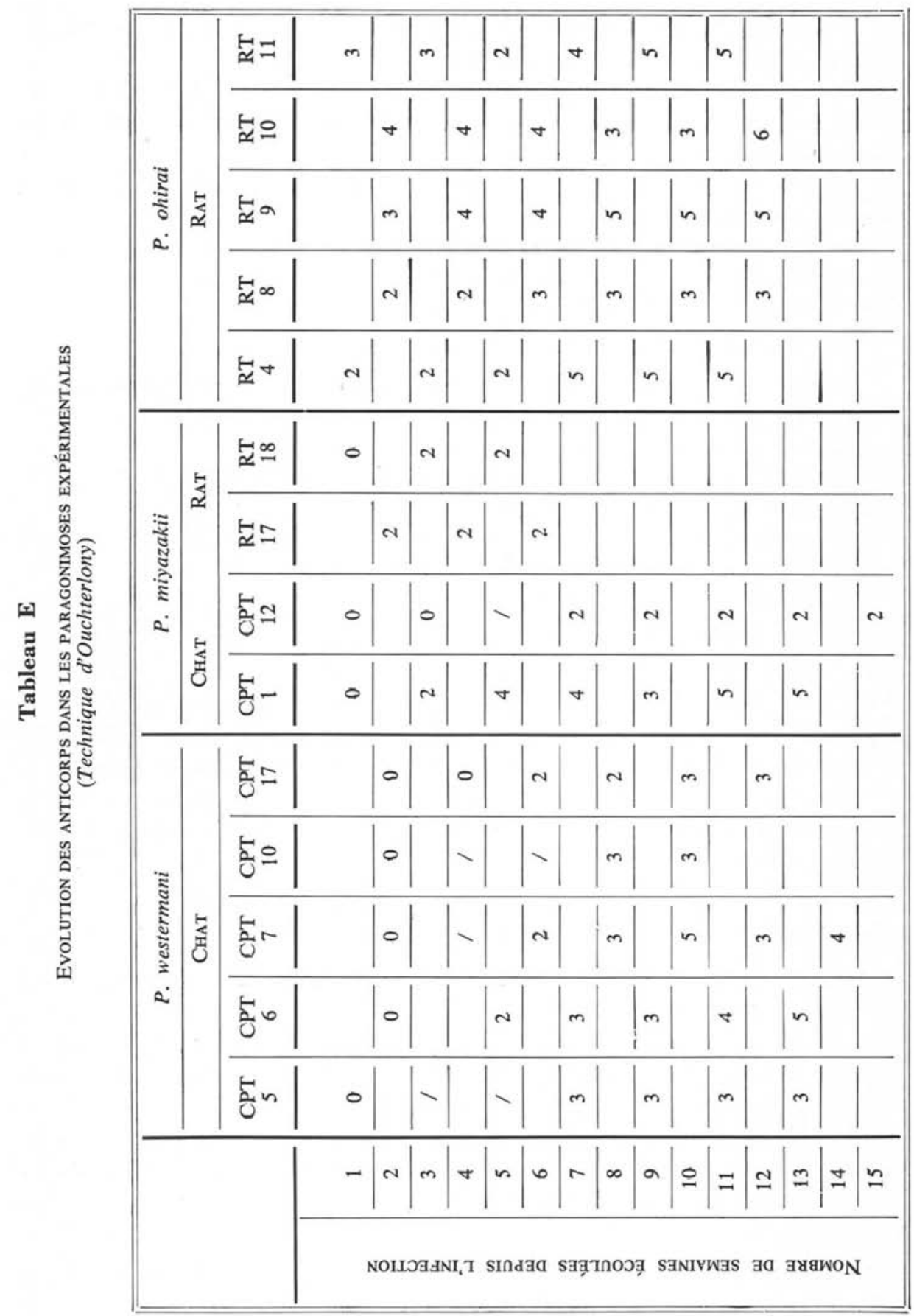




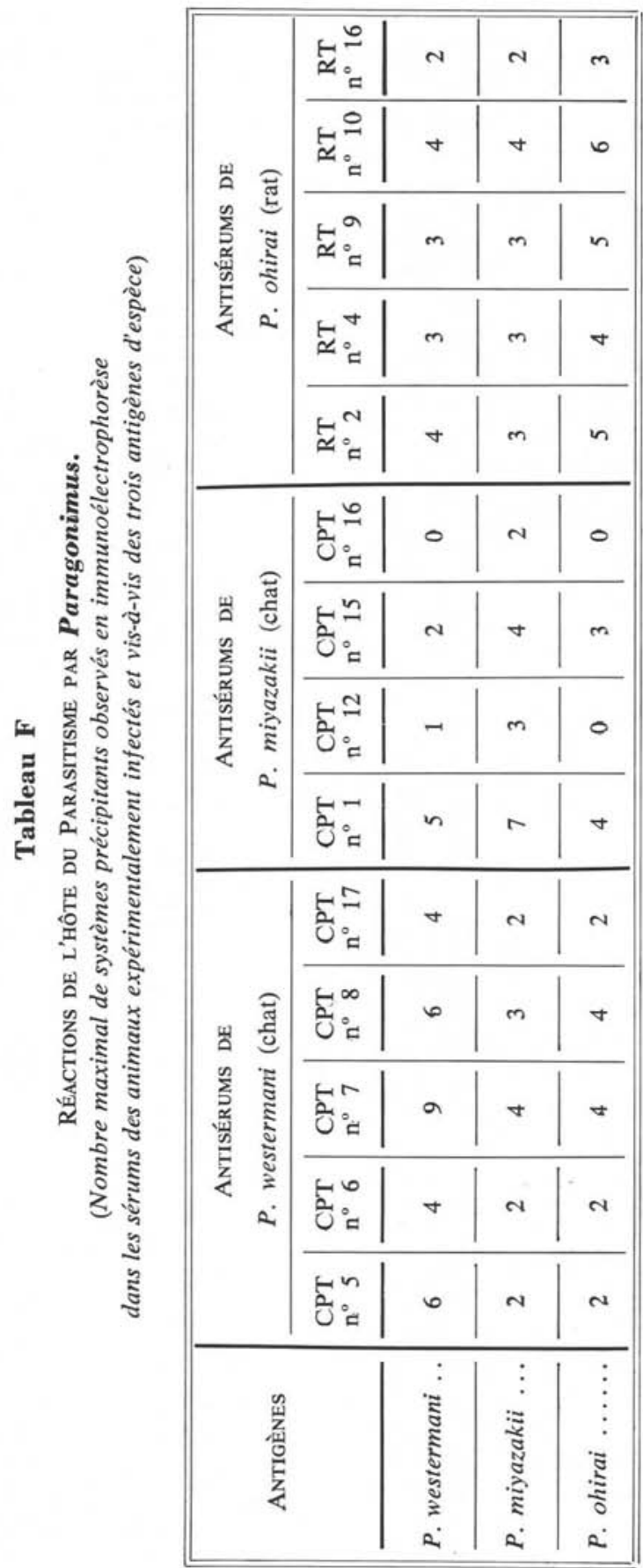


Tableau G.

RÉACTIONS IMMUNOLOGIQUES DE L'HOMME AU PARASITISME PAR

Paragonimus westermani: RÉSULTATS OBSERVÉS EN IMMUNOÉLECTROPHORÈSE AVEC LA RÉACTION DE FIXATION DU COMPLÉMENT ET L'INTRA-DERMO-RÉACTION PRATIQUÉES AVEC L'ANTIGÈNE Paragonimus westermani

\begin{tabular}{|c|c|c|c|c|c|c|c|}
\hline \multirow{2}{*}{$\mathrm{N}^{\circ}$} & \multirow{2}{*}{ I.D.R. } & \multirow{2}{*}{ R.F.C. } & \multirow{2}{*}{ Eufs } & \multicolumn{3}{|c|}{ IMMUNOÉLECTROPHORÈSE } & \multirow{2}{*}{$\begin{array}{l}\text { OUCHTER- } \\
\text { LONY }\end{array}$} \\
\hline & & & & P. westermani & P. miyazakii & P. ohirai & \\
\hline $1 \ldots$ & + & $>\times 160$ & + & 12 & & & 9 \\
\hline $2 \ldots$ & + & $>\times 160$ & + & 10 & 3 & 4 & 4 \\
\hline $3 \ldots$ & + & $>\times 160$ & + & 9 & & & 3 \\
\hline $4 \ldots$ & + & $>\times 160$ & + & 7 & & & 6 \\
\hline $5 \ldots$ & + & $>\times 160$ & + & 5 & 3 & 3 & 6 \\
\hline $6 \ldots$ & + & $>\times 160$ & + & 5 & & & 5 \\
\hline $7 \ldots$ & + & $>\times 160$ & + & 5 & 2 & 2 & 3 \\
\hline $8 \ldots$ & + & $>\times 160$ & + & 5 & & & 3 \\
\hline $9 \ldots$ & + & $>\times 160$ & + & 4 & 2 & 2 & 6 \\
\hline $10 \ldots$ & + & $>\times 160$ & + & 4 & & & 5 \\
\hline $11 \ldots$ & + & $>\times 160$ & + & 4 & 2 & 2 & 4 \\
\hline $12 \ldots$ & + & $>\times 160$ & + & 4 & & & 4 \\
\hline $13 \ldots$ & + & $>\times 160$ & + & 4 & & & 4 \\
\hline $14 \ldots$ & + & $>\times 160$ & + & 4 & & & 3 \\
\hline $15 \ldots$ & + & $>\times 160$ & + & 4 & & & 3 \\
\hline $16 \ldots$ & + & $>\times 160$ & + & 4 & & & 2 \\
\hline $17 \ldots$ & + & $>\times 160$ & + & 3 & & & 3 \\
\hline $18 \ldots$ & + & $\times 138$ & + & 4 & & & 4 \\
\hline $19 \ldots$ & + & $\begin{array}{r}\times \quad 74 \\
\end{array}$ & + & 4 & & & 5 \\
\hline $20 \ldots$ & + & $\begin{array}{r}\times 59 \\
\end{array}$ & + & 7 & & & 6 \\
\hline $21 \ldots$ & + & $\begin{array}{r}\times 52 \\
\end{array}$ & + & 4 & & & 4 \\
\hline $22 \ldots$ & + & $\begin{array}{r}\times 34 \\
\end{array}$ & + & 9 & & & 7 \\
\hline $23 \ldots$ & + & $\begin{array}{r}\times 27 \\
\end{array}$ & + & 2 & & & 2 \\
\hline $24 \ldots$ & + & $\times \quad 21$ & + & 3 & & & 3 \\
\hline $25 \ldots$ & + & $\times \quad 15$ & + & 5 & & & 5 \\
\hline $26 \ldots$ & + & $\times \quad 14$ & + & 2 & & & 2 \\
\hline $27 \ldots$ & + & $\times \quad 12$ & + & 2 & & & 2 \\
\hline $28 \ldots$ & + & nég. & + & 5 & & & 5 \\
\hline
\end{tabular}


Tableau $\mathbf{H}$

COMMUNAUTÉS ANTIGÉNIQUES HôTE-PARASITE

(Réactions minimales observées en immunoélectrophorèse)

\begin{tabular}{|c|c|c|c|c|c|c|c|c|c|}
\hline \multirow[b]{2}{*}{ ANTIGÈNES } & \multicolumn{9}{|c|}{ IMMUNSÉRUMS DE LAPIN ANTI- } \\
\hline & $\begin{array}{l}\dot{5} \\
\triangleq \\
\dot{a}\end{array}$ & 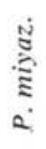 & $\begin{array}{l}0 \\
0 \\
0 \\
0 \\
0 \\
0\end{array}$ & 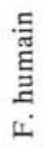 & 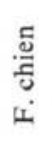 & 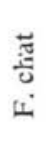 & $\begin{array}{l}\bar{\pi} \\
4\end{array}$ & 离 &  \\
\hline P. westermani ..... & & & & 3 & 4 & 5 & 0 & 4 & 1 \\
\hline P. miyazakii ..... & & & & 2 & 2 & 2 & 1 & 0 & 0 \\
\hline P. ohirai .. $\quad \ldots$. & & & & 0 & 0 & 0 & 7 & 0 & 0 \\
\hline Foiz humain $\ldots$. & 3 & 2 & 0 & & & & & & \\
\hline Foie de chien ... & 4 & 2 & 0 & & & & & & \\
\hline Foie de chat $\ldots . .$. & 5 & 2 & 0 & & & & & & \\
\hline Foie de ra: . & 0 & 1 & 7 & & & & & & \\
\hline Poumon de chat .... & 8 & 0 & 0 & & & & & & \\
\hline Poumon de rat .... & 0 & 0 & 7 & & & & & & \\
\hline S. libertina & 4 & 0 & 0 & & & & & & \\
\hline B. glabrata ......... & 1 & 0 & 0 & & & & & & \\
\hline
\end{tabular}

2. - Communautés antigéniques hôte-parasite (cf. Tableau H).

a) Communautés ANTIGÉniQues AVEC L'Hôte déFINITIF.

Les communautés antigéniques de $P$. westermani avec ses hôtes définitifs sont particulièrement nettes et ce, surtout avec le chat hôte naturel de cette espèce ; elles affectent plus particulièrement la niche écologique du parasite comme le montre la réaction intense obtenue avec le poumon de chat. Par contre, aucune « affinité antigénique » n'est observable avec le rat, animal résistant à l'infection.

A l'inverse, les communautés antigéniques unissant $P$. ohirai et le rat sont aussi évidentes, a ors qu'elles sont inexistantes entre $P$. ohirai et le chat.

$P$. miyazakii semble se comporter d'une façon intermédiaire entre les deux espèces précédentes.

b) Communautés antigéniques aVec L'Hôte intermédiaire.

$P$. westermani possède quatre fractions antigéniques communes avec son hôte intermédiaire naturel Semisulcospira libertina. $P$. miyazakii et $P$. ohirai n'ont par contre aucune parenté antigénique avec ce mollusque chez lequel ils ne peuvent évoluer. 


\section{VI. - COMMENTAIRES}

Les résultats obtenus et leur analyse permettent les commentairés suivants :

a) Sur le plan taxinomique le genre Paragonimus apparaît comme très homogène. Les espèces étudiées présentent cependant de notables différences sur le plan de leur mosaïque antigénique. Les résultats obtenus sont tout à fait superposables à ceux obtenus dans la comparaison du couple Opistorchis felineus et Clonorchis sinensis ou du couple Schistosoma mansoni-Schistosoma japonicum. On n'observe pas d'analogie antigénique presque totale comme entre les espèces Schistosoma mansoni et Schistosoma haematobium ou Fasciola hepatica et Fasciola gigantica, par exemple.

L'analyse de la structure antigénique des trois espèces confirme donc pleinement le bien-fondé de la classification zoologique des Paragonimus.

b) Sur le plan de l'adaptation parasitaire, les communautés antigéniques avec l'hôte définitif et la réponse de celui-ci au parasitisme par les trois espèces étudiées permettent dans les limites de l'expérience réalisée de faire plusieurs remarques:

- $P$. ohirai apparaît comme une espèce bien adaptée au rat, alors que $P$. westermani semble se comporter au contraire comme une espèce plus particulièrement adaptée aux félidés et secondairement à l'homme.

- Les résultats obtenus avec P. miyazakii et confirmant ceux observés par Tada font apparaître ce Troglotrématidé comme une espèce en voie d'adaptation, capable d'évoluer à la fois chez le rat et le chat. $P$. miyazakii possède avec ces deux hôtes des communautés antigéniques faibles, mais présentes dans les deux cas, et suscite chez eux, une réponse immunologique qui est sensiblement de la même intensité.

c) Enfin, sur le plan pratique, il convient de souligner la valeur de l'immunoélectrophorèse dans le diagnostic immunologique de la paragonimose humaine à $P$. westermani, valeur encore accrue par le pouvoir analytique de cetté técnniqué.

\section{CONCLUSION}

L'étude immunologique et plus particulièrement immunoélectrophorétique, des relations hôte-parasite, s'avère être actuellement une bonne méthode d'approche dans la connaissance des phénomènes d'adaptation parasitaire.

Le genre Paragonimus, de par la variété des possibilités biologiques qu'il offre, mérite certainement une étude encore plus approfondie qui serait vraisemblablement en mesure de fournir de précieux renseignements sur les mécanismes d'adaptation parasitaire.

Une telle étude se heurte malheureusement aux difficultés de rassembler le matériel parasitaire nécessaire et oblige la pratique d'expérimentations fractionnées. 
Elle illustre cependant une notion capitale: la connaissance théorique préalable des antigènes parasitaires et de la réponse immunologique de l'hôte expérimental, avant l'application pratique au diagnostic de la maladie humaine des techniques immunologiques.

\section{Bibliographie}

On trouvera de nombreuses références bibliographiques dans les quatre articles suivants : $1-2-3$ et 4 .

1. Capron (A.), Biguet (J.), Vernes (A.) et Afchain (D.), 1968. - Structure antigénique des helminthes. Aspects immunologiques des relations hôte-parasite. Path. Biol., 16, 121.

2. KENT (J. F.), 1963. - Current and potential value of immunodiagnostic tests employing soluble antigens. Amer. J. Hyg., Monographics Series, $\mathrm{n}^{\circ} 22,68$.

3. Yokogawa (S.), Cort (W. W.) and Yokogawa (M.), 1960. - Paragonimus and Paragonimiasis. Exper. Parasitol., 10, 81.

4. Yokogáda (M.), 1965. - Paragonimus and Paragonimiasis. Adv. Parasitol., 3, 99.

5. Abelev (G. I.) et ZvetKov (U. S.), 1960. - Extraction d'un antigène spécifique d'hépatome transplantable de souris par la méthode d'immunofiltration. Voprosyi Onkologyi, 6, 57 (en russe).

6. ANDo (M.), 1917. - Complement fixation test of paragonimiasis. Nihon Byori Gakkai, 7, 633.

7. Biguet (J.), Capron (A.), Tran Van Ky (P.) et d'Haussy (R.), 1962, - Etude immunoélectrophorétique comparée des antigènes de divers helminthes. C.R. Acad. Sci. (Paris), 254, 3600.

8. Biguet (J.), Rose (F.) et Havez (R.), 1960. - Les possibilités d'application de l'immunoélectrophorèse à l'étude des fractions antigéniques des helminthes. C.R. Acad. Sci. (Paris), 251, 982.

9. Biguet (J.), Rose (F.), Capron (A.) et Tran Van Ky (P.), 1965. - Contribution de l'analyse immunoélectrophorétique à la connaissance des antigènes vermineux. Incidences pratiques sur leur standardisation, leur purification et le diagnostic des helminthiases par immunoélectrophorèse. Rev. Immunol., 29, 5.

10. Capron (A.), Yokogawa (M.), Biguet (J.), Tsuji (M.) et Luffau (G.), 1965. - Diagnostic immunologique de la paragonimose humaine. Mise en évidence d'anticorps sériques spécifiques par immunoélectrophorèse. Bull. Soc. Pathol. Exot., 58, 474.

11. Capron (A.), Biguet (J.), Rose (F.) et Vernes (A), 1965. - Les antigènes de Schistosoma mansoni. II. Etude immunoélectrophorétique comparée de divers stades larvaires et des adultes des deux sexes. Aspects immunologiques des relations hôte-parasite de la cercaire et de l'adulte de S. mansoni. Ann. Inst. Pasteur, 109, 798.

12. Grabar (P.) et Williams (C. A.), 1953. - Méthode permettant l'étude conjuguée des propriétés électrophorétiques et immunochimiques d'un mélange de protéines. Application au sérum sanguin. Biochim. Biophys. Acta, 10, 193. 
13. Grabar (P.) et Williams (C. A.), 1955. - Méthode immunoélectrophorétique d'analyse de mélanges de substances antigéniques. Biochim. Biophys. Acta, 17, 67.

14. Headquarters 406th Medical Laboratory, July 1965. - Electrophoretic and serological comparison of lung flukes, Paragonimus westermani, $P$. ohirai, P. iloktsuenensis and P. miyazakii. Professional report, 285.

15. ManoJI (M.), 1952. - Further notes on the lung fluke, Paragonimus ohirai Miyazakii, 1939. II. Immunological studies on the lung fluke, P. ohirai Miyazakii. Igaku Kenhyu, 22, 1197.

16. Ouchterlony (O.), 1948. - Antigen-antibody reactions in gel. Ark. Kemi. Sverige, $26,1$.

17. Phillipson (R. F.) and Mac Fadzean (J. A.), 1962. - Clonorchis, Opistorchis and Paragonimus gel diffusion studies. Trans. Roy. Soc. Trop. Med. Hyg., 56, 13.

18. Sadun (E. H.), Buck (A. H.) and Walton IB. C.), 1959. - The diagnosis of Paragonimiasis westermani using purified antigens in intradermal and complement fixation tests. Mil. Med., 124, 187.

19a. Sawada (T.), Takei (K.) and Yoneyama (K.), 1954. - Studies on the immunodiagnosis of Paragonimiasis. I. The precipitin reaction with crude and fractionated antigens. J. Infect. Dis., 114, 311.

19 b. 1964. - II. Intradermal test with fractionated antigens. J. Inf. Dis., 114, 315.

20. Seed (J. R.), Sogandares-Bernal (F.) and Mills (R. R.), 1966. - Studies on American paragonimiasis. II. Serological observations of infected cats. J. Parasitol., $52,358$.

21. So (N.), 1959. - Immunological studies of the lung fluke Paragonimus ohirai Miyazakii, 1939 (precipitin ring test and Sarles'phenomenon). Fukuoka Med. Acta, 50, 2594.

22. TADA (I), 1967. - Physiological and serological studies of Paragonimus miyazakii infection in rats. J. Parasitol., 53, 292.

23. Tran Van Ky (P.), Tsuji (M.), Capron (A.) and Vaucelle (T.), 1968. - Characterization of the types of enzymic activities in freshly crushed Paragonimus westermani antigens after immunoelectrophoresis in agarose. Hiroshima, J. Med. Sci., 17, 43.

24. Yogore (M. G.), Lewert (R. M.) and Madraso (E. D.), 1965. - Immunodiffusion studies in Paragonimiasis. Amer. J. Trop. Med. Hyg., 14, 586.

25. Yokogawa (M.) and Awano (R.), 1956. - On the complement-fixation test for paragonimiasis. Relation between the intradermal test and the complement fixation test (Japanese). Nihom Izi Shimpo, 27, 1703.

26. Sirol (J.), Kerfelec (J.) et Papinutto (J.-P.), 1967. - La paragonimose pulmonaire en Afrique. A propos de 26 observations colligées au Cameroun occidental. I. Enoncé des faits parasitologiques, anatomo-pathologiques, épidémiologiques et cliniques. Bull. Soc. Pathol. Exot., 60, 533. II. Etude clinique, diagnostique et thérapeutique. Comparaison avec la paragonimose pulmonaire en Asie. Bull. Soc. Pathol. Exot., 60, 543. 